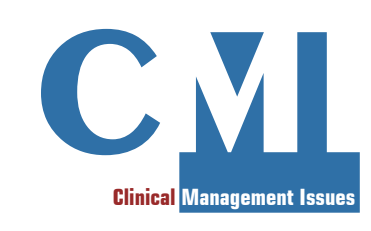

\title{
Acknowledgement to reviewers (June 2016 - June 2017)
}

The editorial staff of Clinical Management Issues (CMI) would like to thank all the reviewers who, with their support and their active cooperation, have contributed to improving the scientific rigor, precision and accuracy of the contents.

- Fabrizio Anniballi

- Filippo Antonini

- Simonetta Bernardini

- Béatrice Bouvard

- Francesco Paolo Cantatore

- Giuseppe Caparrotti

- Simonetta Caria

- Luis José Catoggio

- Andrea Cestari

- Giuseppe Fagone

- Paolo Fascio Pecetto

- Ilaria Izzo

- Vincenzo La Milia

- Marco Lanzillotta
- Pier Luigi Meroni

- Franco Mongini

- Marta Mosca

- Giuseppe Mulè

- Nariman Nawar

- Marica Pecis

- Raffaele Pezzilli

- Matteo Piga

- Maurizio Pompili

- Gian Domenico Sebastiani

- Olivier Sitbon

- Marco Sparaco

- Serena Vita

- Valerio Vizzardi 